\title{
Hospitalised smokers: characteristics, treatment, and transition to ambulatory care
}

\author{
Thomas D MacKenzie
}

Denver Health includes a central hospital with 18000 admissions per year, and a large integrated network of 11 community health centres with 350000 visits per year. Fifty per cent of patients are uninsured, $40 \%$ have incomes below the poverty level, and $70 \%$ are from a minority group.

\section{History of the tobacco control services}

Denver Health has had outpatient cessation classes in the community health centres since the early 1980s. In 1994, we convened a tobacco control committee to address issues such as a tobacco free campus, smoking status assessment as a fifth vital sign, and publication of a tobacco control newsletter. In 1996, when nicotine gum had already gone over the counter (OTC) and nicotine patches were about to become OTC, our payers were deciding not to cover most OTC products. Our challenge was to convince two groups-the hospital pharmacy committee and the Medicaid managed care organisation-that nicotine replacement therapy should be covered. Our approach was to suggest that patches, when used in conjunction with outpatient counselling, are very cost effective-in the order of 10 times more cost effective than screening for hypertension. Knowing that both groups would want to contain costs, we proposed to put the controls on patch distribution listed in table 1. Interestingly, it was in the process of developing these restrictions that we finalised our plans for an inpatient tobacco intervention service (TIS).

\section{Tobacco intervention service}

Once we received approval for inpatient nicotine patches, we proposed to the respiratory therapy department that they would be well suited to serve as the TIS. We

Table 1 Patch distribution controls proposed to pharmacy committee and managed care organisation

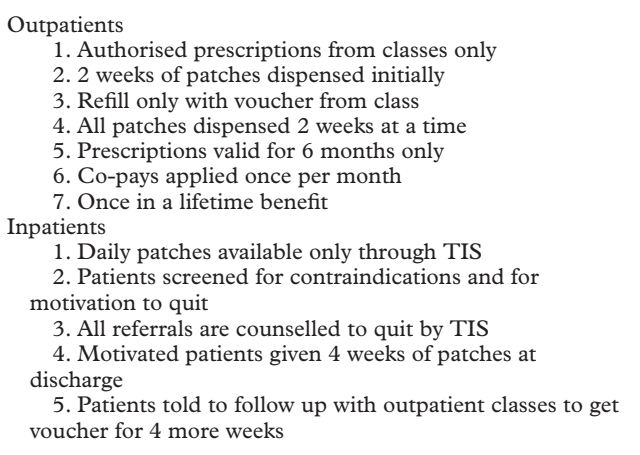

TIS, Tobacco intervention service. created a voice mailbox for referrals to the TIS and posted advertisements on the wards that read "Patches for patients," knowing that the hook for referrals was the restricted availability of nicotine patches. The program has been running successfully for two years. Data on the characteristics of patients who have been treated by our TIS are published elsewhere. ${ }^{12}$

\section{Proposed changes}

We have received some complaints from attending physicians that restricting inpatient patches only to motivated patients ignores the potential benefits of simply treating nicotine withdrawal. They argue that patients might go outside to smoke (and potentially miss scheduled services) less often if they are given nicotine replacement. Thus, we are going to propose to the pharmacy committee that the TIS continue to see all patients going home with patches, but that daily prescriptions for hospitalised patients are unrestricted. Another change we are considering is group counselling for inpatients. Lastly, we are trying to set up a system to increase referrals to the TIS by adding a check box to the standard admission order form for referral to the TIS.

\section{Conclusions}

The successful components of our program are: that it is run through the respiratory therapy department; nicotine patches are a covered benefit; and there is integration of the inpatient and outpatient services. Areas for improvement in the coming years are enhanced smoking cessation services for our employees (especially the respiratory therapists who run the program), a simpler referral process to the TIS, and education for the respiratory therapy staff on counselling techniques.

1 Vernon JD, Crane LA, Prochazka AV, et al. Smokers hospitalized in an urban public hospital: addiction, stages of talized in an urban public hospital: addiction, stages
change, and self-efficacy. Prev Med 1999;28:488-95.

2 MacKenzie TD, Gianani RI. Smoking abstinence after hospitalization: predictors of success. F Gen Intern Med 1999; 14(suppl2):51.

\section{Questions and answers}

Q: Were there any parallel efforts to provide policy changes in the workplace where supportive services to staff who smoked would perhaps catalyse some enthusiasm for participation with patients?

A: We have not done a great job in our institution to address staff smoking. We provide smoking cessation classes at three different sites in our organisation that are open to staff, tmackenz@dhha.org

T D MacKenzie
University of Colorado Health Sciences Center and Denver Health, 1100 Federal 
and are free of charge. Many of our staff members receive their health insurance through our program, thus their primary care providers are in our organisation. They are uncomfortable with attending classes with the patients that they are serving. So, we are now in the process of trying to develop programs that are targeted to the staff. Additionally, nicotine patches are a covered benefit for our employees. They receive eight weeks of patches as long as they are enrolled in counselling. We provide our employees with the same benefits as patients. We have a smoke free campus and we have become more and more strict in enforcement. We have a few zealots in our organisation who, when they see a staff member smoking outside of the hospital, will come down on them quite hard. I am not sure that is the right approach, however. We need to construct better programs; we have not done a great job of addressing staff smoking in our hospital.

Q: You said that in your system nicotine patches were considered a once-per-lifetime benefit. Does that mean that you perform screening to determine where the patient is in terms of stage of change in order to maximise that one chance?

A: That is a great question. Initially we thought that we would only target those patients who were not in the precontemplation phase and that we would provide patches only for them. I think the data are somewhat mixed in terms of the success of the patch by stage of change, particularly in the outpatient population. Thus, we decided not to make any restrictions. It is difficult, however, because it is essentially a cost-control measure. As we all know, people aren't always successful in their attempt at quitting. I have had a lot of patients who have stated that they weren't quite ready when they signed up for classes and received the patches. Now they're ready, and they still have a couple of boxes of patches and want to re-enroll. I don't know what the answer is. I don't know whether I should advocate for providing a second course for certain people or not. I am not sure how cost effective a second course of nicotine patches would be in the population that we serve. 